\title{
Oral Cancer Surgery and COVID 19 Pandemic: Our Experience
}

\author{
Kantharia $\mathrm{S}^{1 *}$, Kantharia $\mathrm{RA}^{2}$ and Reddy $\mathrm{P}^{3}$ \\ ${ }^{1}$ Consultant ENT and Head and Neck Surgeon, Kailash Cancer Hospital and Research Centre, \\ India
}

${ }^{2}$ Consultant Head and Neck Surgeon, Kailash Cancer Hospital and Research Centre, India

${ }^{3}$ Fellow, Head and Neck Oncosurgery, Kailash Cancer Hospital and Research Centre, India

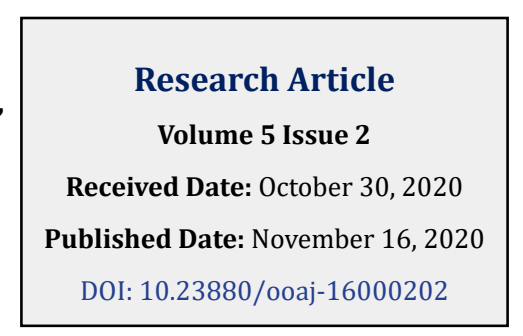

*Corresponding author: Shehnaz Kantharia, Consultant ENT and Head and Neck Surgeon, Kailash Cancer Hospital and Research Centre, Gujarat, India, Tel: +91-9537511001; Email: shehnaz.kantharia@greenashram.org

\section{Abstract}

The COVID 19 pandemic limited the clinical practice of Surgical Oncologists all over the world to performing emergency procedures and surgeries. During this initial period of two to three weeks we realised that further delay in the treatment of curable oral cancers would have serious adverse prognostic implications. So we started the process to formulate our own institutional guidelines to screen and test all preoperative asymptomatic oral cancer patients for COVID 19. All preoperative oral cancer patients were tested for Reverse Transcriptase Polymerase Chain Reaction (RT PCR) and a negative COVID RT PCR report was followed by HRCT Chest. We share our prospectively collected data of Oral Cancer Surgery patients from 14th April to 31st May 2020 with details of sub site, stage, operative procedure and type of reconstruction offered. Our institutional protocol to test all preoperative patients by the highly sensitive HRCT Chest combined with the gold standard RT PCR assay ensured the safety of our patients, attendants and the hospital staff.

Keywords: COVID-19; Oral Cancer Surgery; CO-RADS; RT-PCR; HRCT Chest; Guidelines; Permission

Abbreviations: COVID-19: Corona Virus Disease-2019; ICMR: Indian Council of Medical Research; CDHO: Chief District Health Officer; RSI: Rapid Sequence Induction; RTPCR: Reverse Transcriptase Polymerase Chain Reaction; HPV: Human Papilloma Virus.

\section{Introduction}

Globally most countries were hit by the Corona Virus Disease-2019 (COVID-19) which within no time reached pandemic proportions and took all of us by surprise. In India, while the initial rise in the number of cases from January to March was slow and steady, it was by the 3rd week of March that the Government of India and Health authorities became aware of the magnitude of the problem and decided to enforce a lockdown. By the end of March 2020, the number of positive cases almost reached 1000 with 25 deaths.

Worldwide even the best healthcare facilities and medical infrastructure had a hard time managing patients during its peak. The rapid rate of transmission of COVID 19 has impacted every facet of our global and domestic societies and has left the entire healthcare system struggling to manage the increasing number of positive cases.

As Clinicians working in a speciality cancer hospital, we quickly started getting familiar to handling an infection pandemic along with providing compassionate, high quality care under circumstances we have never had to face before. As frontline warriors we knew that our fight was against two dreaded C's - CANCER \& COVID, but as usual we will have to continue to tame it with more COURAGE \& COMPASSION. 


\section{Otolaryngology Open Access Journal}

Technology helped us tremendously in reaching out to our patients and to communicate with our colleagues in other cancer institutes for their inputs and strategies. Realizing the importance of physical distancing, our face to face meetings were replaced by interaction on WhatsApp, Telegram and webinars on Zoom.

Our hospital is a leading cancer hospital of India and the second largest comprehensive cancer centre of Gujarat providing treatmentand opinions to around 25,000 new cases annually with over $30 \%$ of patients coming from outside the state of Gujarat. Our Institute is located in a rural-semi urban setting of western India catering to patients from Gujarat and the neighbouring states. Most of our patients belong to a poor socioeconomic status and are predominantly daily wage earners with a low level of education. Logistics of travel and stay of the patients and their relatives was a matter of serious concern while following the general principles of social distancing [1-3].

Amidst the COVID 19 crisis, we decided to continue treating our patients across all the specialties- Surgical, Medical \& Radiation Oncology and to run our Bone Marrow Transplant program. Protection of our staff, judicious use of available resources, and a rational and balanced attitude towards cancer patients was the need of the hour. As our first step we set up a task force which included clinicians, matrons, nursing staff, paramedics and hospital administration. The work assigned to them was to see to it that the general guidelines issued by the government are followed, departmental strategy is adhered to and to ensure proper liaison between the state health authorities and the hospital. It was difficult initially as the government decisions often changed on day to day basis requiring us to alter our hospital guidelines, but with continuous employee education, staff safety and communication with the state health officials we prepared ourselves to navigate through these difficult times.

Unfortunately, COVID 19 has limited the clinical practice of different medical specialties with some of them still struggling to resume in a phased manner. As Oncologists, our priority was to strike a balance between the need to continue cancer treatment and to ensure safety of patients and employees.

At our hospital Head \& Neck Cancers form about 50\% of our patient load of which $80 \%$ are ORAL CANCERS. We treat around 10000 oral cancer patients annually of which 3000 are newly diagnosed patients and around 1000patients requiring curative surgery with a waiting list of seven to ten days. In the beginning like everyone else we were overwhelmed by the deluge of information on COVID-19. There were guidelines being made and disseminated by various Medical Association and Institutes with conflicting views and recommendations. However, everyone agreed that all aerosol generating procedures or surgeries involving the Oral Cavity, Nasal Cavity, Nasopharynx, Oropharynx, Trachea and Bronchus carried a very high VIRAL LOAD and had high risk of transmission of the Corona virus if the patient was infected with it $[4,5]$.

The Indian Council of Medical Research (ICMR) released a guideline for COVID testing on $17^{\text {th }}$ March 2020. They said that the test must be done in

a) Asymptomatic people who had undertaken air travel

b) Contacts of people who had tested positive for the virus and

c) Health care workers involved in care of patients with respiratory distress/severe respiratory illness when they became symptomatic.

There was no mention or provision for asymptomatic patients to be tested prior to undergoing a major surgery, especially something as critical as Oral Cancer Surgery where deferring treatment for a couple of weeks could lead to progression of disease and advancement of the stage rendering an operable disease inoperable.

The first few days went in counselling the patients and their relation and deferring all elective surgeries. Few lifesaving emergency procedures like tracheostomy were performed donning full PPE which was a novel experience in itself. We started Neo adjuvant Chemotherapy and Oral Metronomic Chemotherapy in some patients having advanced Oral cancers after approval from a Virtual Tumour Board. We read all guidelines, attended various webinars and tried to learn how to continue treating our cancer patients through the pandemic. We realized that the best possible way to ensure safety of patient, relation, our staff and ourselves would be to do a COVID-19 RT PCR test for all asymptomatic pre-operative Oral Cancer patients.

The guidelines still said that asymptomatic patients were not to be tested. We contacted the Chief District Health Officer (CDHO) of Vadodara and explained our proposal. He was extremely co-operative, understood our quandary and directed us to the Chief District Medical Officer. He was extremely co-operative and after consulting the Health Commissioner of Gujarat, they gave our centre permission to carry out COVID-19 testing in patients having Oral Squamous Cell Carcinoma who were operable and asymptomatic for Covid-19. This permission was first of its kind to be given to an Institute by the State Government for testing asymptomatic patients.

We started admitting operable Oral Squamous Cell Carcinoma patients from $15^{\text {th }}$ April onwards and started 
operating them from $18^{\text {th }}$ April till $1^{\text {st }}$ June during the nationwide lockdown. In a span of 45 days we operated 46 patients after getting an RT PCR (Reverse Transcriptase Polymerase Chain Reaction) test for COVID-19.

\section{Material and Methods}

On day one patient would be admitted in an isolated special room. Within an hour of admission Nasopharyngeal and Oropharyngeal swab would be collected. Collection was done using full PPE, N-95 mask and face shields by the testing doctors. The collected swabs would then be transported as per specifications from the testing laboratory. On day two once the COVID-19 negative report was received, patient would then be sent for HRCT of Chest. Our Radiologist reported theses scans on basis of CO-RADS (Figures $1 \& 2$ ).

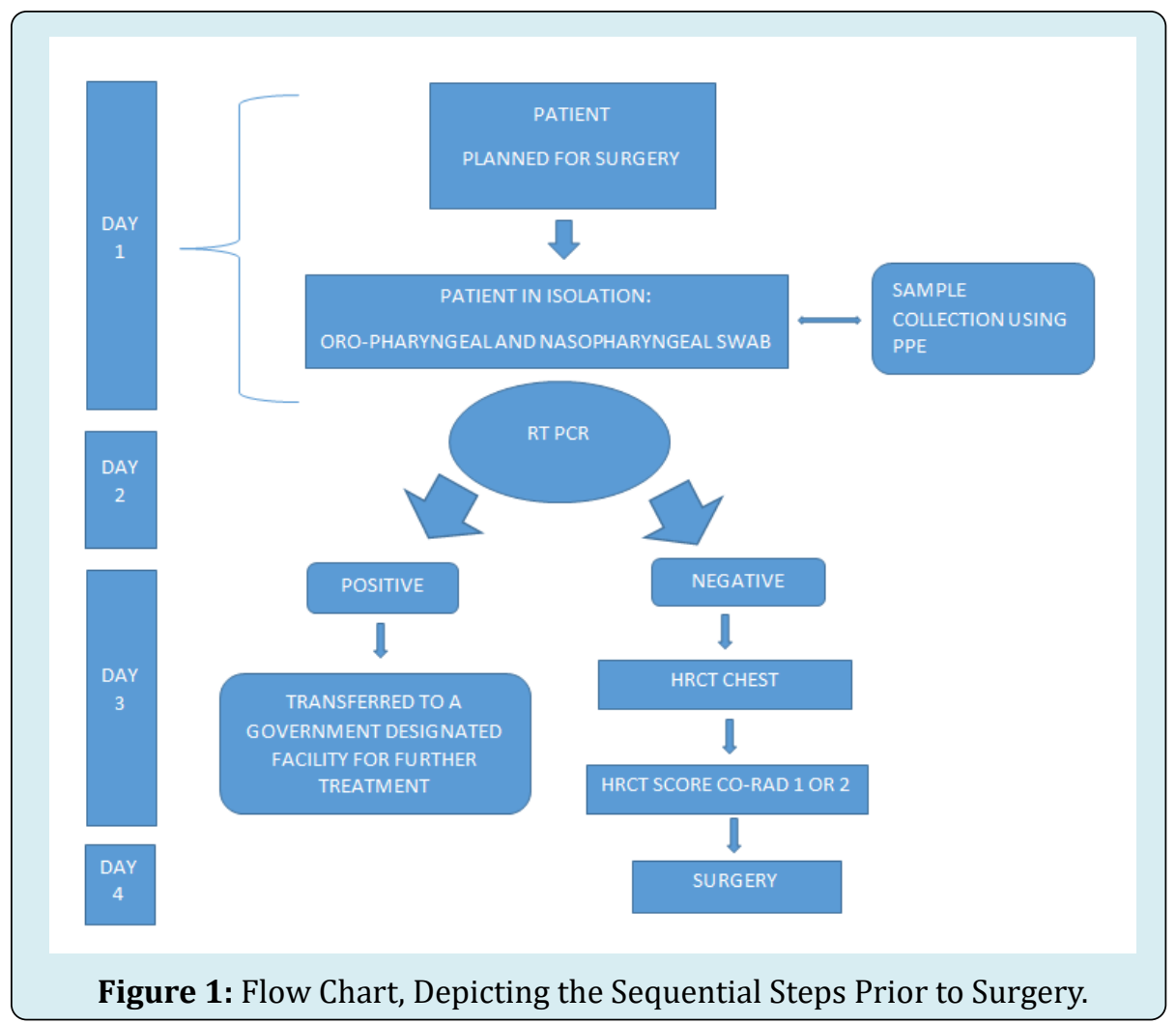

Figure 1: Flow Chart, Depicting the Sequential Steps Prior to Surgery.

\begin{tabular}{|l|c|c|}
\hline & \multicolumn{2}{c|}{ CO-RADS* } \\
Level of suspicion COVID-19 infection
\end{tabular}




\section{Otolaryngology Open Access Journal}

Based on the CT findings, the level of suspicion of COVID-19 infection is graded from very low or CO-RADS 1 up to very high or CO-RADS 5 and the severity and stage of the disease are determined with remarks on comorbidity and a differential diagnosis [6-8].

All patients who underwent surgery had a RT PCR negative and a no or low level of suspicion on HRCT Chest. During surgery our team of Anaesthesiologists followed all the guidelines as prescribed by the All India Difficult Airway Association Consensus Guidelines for Airway Management in the operating room during COVID-19 pandemic.

As per the guidelines, all spontaneously breathing patients should wear a surgical face mask. They should be directly transferred to the OR bypassing the preoperative holding area. Patient examination, including airway evaluation, should be performed using full PPE. The patients should be wearing a surgical mask and covered with a transparent plastic sheet or a COVID box on the Operating Room table. Rapid sequence induction (RSI) is the preferred method to avoid mask ventilation and facilitate faster Tracheal Intubation. Video assisted laryngoscopy is recommended for Tracheal Intubation.

Single use items were immediately discarded in the appropriate disposal bag and reusable items dropped in to the container with disinfectant solution without touching them elsewhere, to prevent contamination of the OR and health care workers. Although we operated only on preoperatively confirmed COVID negative patients, all necessary precautions were taken during and after surgery by the Operating Surgeons, Head \& Neck Fellows, OT assistants, Nursing staff and housekeeping staff.

\section{Results and Analysis}

From $18^{\text {th }}$ April 2020 till $31^{\text {st }}$ May during the nationwide lockdown we operated 46 patients of Oral Squamous Cell Carcinoma. There were 36 Males and 10 females in the age group of 25-65 years. Three patients with Hypertension and Diabetes Mellitus as co-morbidities were put on treatment and maintained by physician. 15 patients had early stage (T1-T2) and 31 had late stage (T3-T4) Oral Squamous Cancer. 30 cases had Buccal mucosa as the primary site of Cancer, 1 case had Synchronous primary of Buccal mucosa and Papillary carcinoma of Thyroid Bethesda category V, 8 cases of Squamous carcinoma of Tongue, 3 cases had cancer of Mandibular alveolus, 2 patients had upper gingivobuccal sulcus as primary site and there were 2 cases of floor of mouth Squamous cell carcinoma. Out of these 46 cases, 7 were recurrence of squamous cell carcinoma within 2 years of treatment of primary disease and 4 cases were post 2 cycles of Neo Adjuvant Chemotherapy. Two patients who tested positive for COVID-19 were immediately transferred to the Government designated COVID hospital in Baroda immediately for further management of COVID.

\section{Discussion}

Oral cancer is among the top three cancers in India and is one of the leading cause of death in adults. The incidence of oral cancer is highest in India, south and Southeast Asian countries. In India, 20 per 100000 populations are affected by oral cancer which accounts for about $30 \%$ of all types of cancer and over 5 people in India die every hour every day because of oral cancer. Oral cancer is any malignant neoplasm which is found on the lip, floor of the mouth, cheek lining, gingiva, palate or the tongue. Alcoholism, tobacco usage in the form of cigarettes, chewed tobacco, betel nut and human papilloma virus (HPV) are the most common risk factors for oral cancer. Oral cancer may also occur due to poor dental care and poor diet. In India, $90-95 \%$ of the oral cancers is squamous cell carcinoma.

Our hospital is a tertiary care cancer hospital treating around 15000 new oral cancer patients annually. After the nationwide lockdown happened, as oncologists our priority was to strike a balance between the need to continue cancer treatment and to ensure safety of patients and employees. Our challenge was to timely treat the oral cancer with a curative intent knowing that oral cavity has the highest viral load of COVID 19. As during the initial two weeks of the lockdown, there were no clear instructions from the State Health Department, we prepared a proposal for preoperative testing of asymptomatic oral cancer patients and submitted it to the health officials. Once our proposal was approved by the Chief District Health Officer and Chief District Medical Officer, we starting testing of all asymptomatic preoperative Oral Cancer patients for COVID-19 before operating them. In this way we started treating our patients during the lockdown and also ensured safety of our patients and hospital staff. Also while treating these patients it was extremely important to use our discretion to ensure a proper case selection so as to justify the use of the available setup (hospital beds and ventilators), manpower, PPE, blood and blood products.

\section{Conclusion}

As surgical oncologists working in a high volume cancer centre it is important to develop our own institutional protocols for prioritizing surgical treatment after taking into account all the ground realities amidst the pandemic. Our commitment to our patients to treat operable squamous carcinoma of oral cavity spurred us to convince the government authorities to allow for COVID-19 testing for asymptomatic patients before operating them, in the early days of the pandemic and subsequent lockdown. We are 


\section{Otolaryngology Open Access Journal}

happy to state that we paved the way for other Oncologists across Gujarat to do the same and also for the State Government to revise its guidelines for COVID-19 testing in asymptomatic patients.

\section{References}

1. Pramesh CS, Badwe RA (2020) Cancer Management in India during COVID-19. N Engl J Med.

2. HodelRio C, Malani PN (2020) COVID-19 new insights on a rapidly changing epidemic. JAMA 323(14): 1339-1340.

3. WHO (2018) Latest Global Cancer Data: Cancer Burden Rises to 18.1 Million New Cases and 9.6 Million Cancer Deaths in 2018. Geneva, Switzerland.

4. Guidance Document on Appropriate Management of Suspect/Confirmed Cases of COVID-19. New Delhi, India:
Ministry of Health and Family Welfare.

5. Ferlay J, Colombet M, Soerjomataram I, Mathers C, Parkin DM, et al. (2019) Estimating the global cancer incidence and mortality in 2018: GLOBOCAN sources and methods. Int J Cancer 144(8): 1941-1953.

6. WHO (2020) Coronavirus Disease 2019 (COVID-19) Situation Report-85. Geneva, Switzerland.

7. Prokop M, Everdingen W, Vellinga TR, Ufford HQ, Stoger L, et al. (2020) CO-RADS A categorical CT assessment scheme for patients suspected of having COVID-19: definition and evaluation. Radiology 296(2): 97-104.

8. Patwa A, Shah A, Garg R, Divatia JV, Kundra P, et al. (2020) All India difficult airway association (AIDAA) consensus guidelines for airway management in the operating room during the COVID-19 pandemic. Indian J Anaesth 64(14): 107-115. 